\title{
1. Las aspiraciones distributivas de la justicia global
}

\author{
Iván Vargas-Chaves ${ }^{1}$
}

\section{Introducción}

La justicia global se da en un escenario en el que el orden global institucional viene siendo cuestionado porque no logra una armonización adecuada entre los intereses político-económicos y los intereses humanos fundamentales (Miller, 2007). Desde este escenario crítico, se llevan a cabo reflexiones que dirigen la propuesta que Pogge (2001) hace de 'lo moral', como eslabón clave para la consecución de una sociedad justa y equitativa, en el potencial que este novedoso enfoque de justicia tiene para romper cualquier tensión generada desde las dinámicas entre el derecho interno, supra e internacional.

De hecho, el concepto de justicia global ha logrado desmarcarse de la justicia supranacional e internacional. La diferencia yace en el fin perseguido y en lo que se entiende por justicia. Así, en la justicia supranacional e internacional es el Estado el que se encarga de generar las dinámicas para la consecución de los fines de lo que -institucional e históricamente- se concibe como justicia.

En la justicia global, por el contrario, no es relevante la distinción entre las diversas definiciones de justicia entre estados o naciones; como sí en la labor de adentrarse en el caparazón del estado -o state shell- e indagar en qué consiste realmente la justicia para los seres humanos. (Brock, 2017). Ello, sin sacrificar la labor de descifrar las conexiones entre los principales problemas abordados, a través de los dilemas que la globalización les plantea a las instituciones desde una orientación moral e interactiva (Grupo de Investigación en Justicia Global, 2017). En todo caso,

1 Abogado de la Universidad del Rosario. Máster en Derecho de la Universidad de Génova, Italia. Máster en Derecho Privado y Doctor en Derecho de la Universidad de Barcelona, España. Dottore di Ricerca (Dott. Ric.) de la Universidad de Palermo, Italia. Docente investigador de la Corporación Unversitaria del Caribe - CECAR. 
lo que verdaderamente importa es encontrar el mejor lugar para la justicia en una escala global, a través de 'eslabones'2 que permitan comprender cuáles son las responsabilidades que tenemos como seres humanos. (Risse, 2012; Mandle, 2006).

Uno de los principales referentes para los estudiosos de la justicia global es la obra El Derecho de Gentes de John Rawls. Desde esta propuesta ha surgido una importante corriente de pensamiento que, estimulada e influida por los postulados de este influyente teórico, empezó a repensar los modelos de justicia en dirección a una justicia global. Desde entonces varias preguntas se han vuelto claves en las discusiones, como por ejemplo ¿Qué responsabilidades tenemos con los pobres en el contexto global? ¿Debería la desigualdad global ser moralmente preocupante? ¿Cómo podríamos hacer una transición efectiva hacia un mundo menos injusto? (Brock, 2017).

El presente texto constituye un esfuerzo por llevar a cabo un acercamiento teórico a una de las grandes cuestiones de la justicia global: la pobreza en el mundo. Para lograrlo, analizamos esta problemática desde distintas aproximaciones en las que se intenta reflejar las aspiraciones distributivas de la justicia global mediante tres visiones sobre la pobreza.

En primer lugar, afrontaremos el concepto y la medición de la pobreza, que de antemano señalamos requiere de enunciaciones amplias y multidimensionales, a fin de diseñar estrategias para que las vías de cooperación puedan responder apropiadamente a las necesidades específicas.

En segundo lugar, señalamos algunos de los grandes retos que trae consigo el binomio pobreza-diversidad en un contexto global. Y, por último, delinearemos el concepto del deber moral de asistencia desde los deberes correlativos, con miras a plantear unas reflexiones finales que sinteticen los puntos relevantes de este artículo.

2 Algunos de estos eslabones plantean retos en (i.) La identificación de lo que debería contar como problemas importantes de justicia global planteando soluciones a cada problema identificado; (ii.) La identificación de quién o quiénes podrían tener responsabilidades al abordar el problema identificado; (iii.) La argumentación de posiciones sobre qué agentes particulares deberían hacer en relación con la solución de cada problema; y (iv.) El proporcionar un enfoque jurídico que fundamente lo anterior. (Brock, 2017). 
El problema de la pobreza desde la justicia global, más allá de ser un asunto de distribución de los recursos como lo conciben algunos teóricos, es una cuestión que debe solventarse desde enfoques que solucionen el desconocimiento de elementos estructurales; siendo algunos de estos (i.) Su carácter multidimensional, (ii.) La diversidad y (iii.) El alcance real que debe tener el deber moral de asistencia.

Además, si bien es cierto que el mundo nunca antes fue tan pobre, ciertamente tampoco logró ser tan rico como ahora. De ahí la necesidad de redirigir las estrategias de lucha contra este problema. A la par de esta realidad, somos conscientes de que el crecimiento económico y la globalización, sin políticas sociales y económicas nacionales estratégicamente dirigidas abrirá aún más las brechas de la desigualdad.

Sin lugar a dudas, no pretendemos dar una solución al problema, sino ilustrar algunas ideas que puedan aportarse al proceso de buscar salidas favorables. Tampoco podemos negar que otros factores son igualmente necesarios para mitigar este flagelo, como lo son la corrupción, las limitantes para el ejercicio y goce del derecho humano a la transparencia y, por supuesto, el diseño y activación de políticas de inclusión social necesarias para cerrar la puerta a todo tipo de discriminación.

\section{El carácter multidimensional de la pobreza}

Siendo la pobreza un concepto tan complejo, mal haríamos en reducirla a un único significado. Su carácter multidimensional en el que convergen diferentes aspectos de la vida humana, ha permitido que sea estudiada desde diferentes enfoques y disciplinas. ${ }^{3}$ Boltvinik \& HernándezLaos (1999, p. 29-30) al abordar esta problemática, parten de las acepciones de necesidad, carencia, y de lo indispensable para el sustento de la vida.

Estas necesidades no satisfechas, encuentran eco en los significados de pobreza identificados por Spicker (2009, p. 292), quien sostiene que la pobreza es entendida en, al menos, doce sentidos específicos, superpuestos

3 Son numerosas las definiciones de pobreza que se pueden encontrar. Algunas de las más aceptadas podemos estudiarlas en Miller \& Roby, 1967; Ashton, 1987; Baratz \& Grigsby, 1971. 
unos sobre otros, y de los cuales, dos o tres definiciones pueden hallarse simultáneamente en una misma posición en el debate sobre la pobreza.

No obstante, es preciso tener presente que no toda necesidad debe ineludiblemente derivar en pobreza, tal como se ha entendido desde la óptica de la justicia global. Ello, además, implica que las políticas públicas deben enmarcarse en darle prioridad a tales necesidades, determinando cuáles se vincularían al concepto, por ejemplo, sobre la alimentación y el acceso a la salud y a la educación.

La forma más clara de referirnos a la pobreza es, sin duda, la situación económica, como consecuencia de unos ingresos bajos. A manera de juicio moral (Cf. Altimir, 1981), y, desde el punto de vista social como una condición de clase, que es excluyente y que sitúa al grupo de personas desfavorecidas en una situación de dependencia.

A pesar de ser plausible que todas las definiciones sobre la pobreza se encuentren simultáneamente en una misma discusión, no siempre estas pueden ser aplicadas en todos los contextos. Por lo demás, es importante hacer un énfasis en que, hoy por hoy, los estudios sobre pobreza no apartan su atención de aspectos tales como la necesidad, el estándar de vida, y la insuficiencia de recursos (Ribotta, 2011; Spicker, 2009).

En relación a la insuficiencia de recursos ${ }^{4}$, a partir de la relación entre el ingreso y el consumo, este suele ser quizás el enfoque más utilizado a nivel internacional para medir la pobreza, dado su alcance como la incapacidad para alcanzar un nivel de vida mínimo; y en el que interfieren dos elementos básicos. Por una parte, es importante saber el gasto necesario

4 El Banco Mundial valoró el gasto según la paridad del poder adquisitivo o PPA. En una línea de tiempo: (i.) para 1991 la línea de pobreza internacional era equivalente a 1 dólar PPA diario; (ii.) Para el año 2000 se recalculó la cifra, fijándose en 1,08 dólares diarios; (iii.) En el 2005 se determinó un nuevo umbral, quedando en 1,25 dólares PPA diarios. Por lo tanto, la línea elaborada por el Banco Mundial para la pobreza extrema se ubica en el margen de un dólar diario. Se trata de un enfoque basado en la renta, el cual no necesariamente debe rechazarse aunque sí debe ser observado críticamente, pues ante un aumento de la desigualdad de ingresos, quienes se encuentren en el umbral de la pobreza, no podrán participar adecuadamente del crecimiento económico de su país, una vez que este llegue (PNUD, 2003). Por tanto, el gobierno de turno no contará con las estadísticas adecuadas para implementar un plan óptimo de distribución, toda vez que quedan por fuera otros indicadores sumamente importantes, como son la mortalidad infantil, el analfabetismo, el acceso a la salud, la educación y la vivienda adecuada que forman parte de ese nivel mínimo o básico de vida, que todo ser humano tiene derecho a gozar. 
para acceder a un nivel de necesidades muy básicas, y de la otra, los indicadores cuantitativos que pretenden reflejar el costo de la participación en la vida diaria de las sociedades (Banco Mundial, 1990).

En contraposición a este enfoque, el Programa de la Naciones Unidas para el Desarrollo, PNUD, en su informe sobre desarrollo humano de 1996, hace un llamado de atención e introduce un nuevo índice ${ }^{5}$ basado en las capacidades, a partir de una medición de la pobreza más centrada en el ser humano. Con ello, se pretende alcanzar el reconocimiento de las privaciones humanas desde varias esferas críticas, siendo la falta de ingresos tan sólo una de esas esferas (Fraser, 2008, p. 84).

La importancia de este último enfoque yace en la privación de las capacidades básicas, esto es, en el desarrollo humano, y no exclusivamente -como se enfatiza también en los estudios sobre justicia global- en la ausencia de ingresos. Es, de acuerdo a Sen (2000, p. 117), un factor que impide el desarrollo de las capacidades de las personas, y quizás la privación mayor, pues suprime las razones que tiene el ser humano para perseguir las libertades necesarias para poder satisfacer sus fines.

Creemos pues, que la contribución de tal enfoque en la reducción de toda brecha, encuentra su punto de partida en la compresión holística de la pobreza, en tanto sus causas y naturaleza parten de privaciones intrínsecamente importantes; contrario al enfoque de ingreso, meramente instrumental e institucional, siguiendo la línea que Pogge (2001) traza en 'Priorities of global justice'.

Primero hay que sostener que la renta no es el único factor que genera o priva a las personas de capacidades, y segundo, la relación entre la ausencia de renta y falta de capacidades -insistimos- es instrumental, pudiendo existir variaciones entre comunidades, familias e incluso individuos. De allí, nuestro interés en analizar a continuación la pobreza, desde la óptica del desarrollo global y humano.

Al margen de lo analizado hasta ahora, el concepto de desarrollo, al igual que el de pobreza, no cuenta con una definición unificada. Su noción o significado ha pasado por diversas adaptaciones históricas, en el que las

5 El índice de pobreza de capacidad, o IPC, un índice que en el concepto del propio Programa de Naciones Unidas para el Desarrollo, aborda una medición desde una óptica multidimensional, aunque centrada, en todo momento, en la capacidad humana. 
teorías han ido evolucionado constantemente. (Cf. Hine \& Montiel, 1999, pp. 958-959; Pinazo, Peris \& Gámez, 2010, p. 393; Rist, 2002) Es por esto que creemos que lo más apropiado es apartarnos de la pobreza como un concepto, para concebirla como el fin mismo del desarrollo.

La medida del desarrollo, al igual que el de la pobreza, se ha concentrado en cifras que valoran el ingreso, y lo que debería ser un medio se convierte en un fin: el crecimiento económico ${ }^{6}$. No obstante, los ingresos no garantizan por si mismos la reducción de otro grupo de carencias necesarias para alcanzar un nivel de vida adecuado. En este aspecto radica la importancia de prestar un mayor cuidado a esa estrecha relación entre el desarrollo y la pobreza.

Cuando nos referimos al desarrollo desde una perspectiva de pobreza, ineludiblemente debemos hacer referencia a los derechos contenidos en el Pacto de derechos Económicos Sociales y Culturales, de los que a su vez se valen las teorías de la justicia que incorporadas a este contexto, deben partir de un sustento primario de derechos civiles y políticos o en términos de Rawls (1982, p. 159) de los bienes primarios.

Por añadidura, toda reivindicación individual no habrá de valorarse siempre en términos de bienes primarios, cómo sí en términos de las libertades que los ciudadanos tendrían para elegir su propio modo de vida. ${ }^{7}$ Para Sen (1998, p. 115) esta es la capacidad humana. Al respecto Pogge (2005, p. 68) es enfático en sostener que la mejor manera de ejercer estos derechos, recae en la exigibilidad de mecanismos que impidan la concentración de la propiedad de la tierra, la usura o el acaparamiento especulativo de los artículos de primera necesidad; que fomenten la

6 La repercusión del crecimiento económico, si bien tiene un efecto indirecto, que permite inversión en educación, salud y otras estructuras básicas, es indirecto, ya que su fin no busca alcanzar tal resultado, lo que implica que puede darse o no. Es una cuestión de azar y, finalmente, el goce de tales derechos depende de la buena suerte o, por lo menos, de otros factores externos.

7 Debemos aquí pensar, por otra parte, que el igualitarismo en Lukes (1993, pp. 19-40) lo que busca es una construcción comunitaria, y no trata de abordar tales problemáticas asignándoles a cada individuo y a cada comunidad local un único status, salvaguardado a través del aparato estatal. (Cante, 2010, p. 91) Aunque, no obstante, reconozca que si fuese un ideal, no es una actitud natural en la sociedad en la que estamos inmersos. En cualquier caso, la demanda de un igualitarismo es un reclamo de esta sociedad al quedar en duda los principios de distribución justa e igualdad. 
educación, especialmente, desde sus primeras etapas, y, que prevean subvenciones, subsidios y prestaciones.

Dower (1995, pp. 377-390), por su parte, cree que la verdadera cuestión yace en la asistencia del desarrollo, por lo tanto, se debe partir del concepto de desarrollo que no tiene nada que ver con la idea de crecimiento económico. Puesto que el crecimiento económico no es capaz de reflejar modelos occidentales de los cambios que deberían tener lugar en los países pobres y no-occidentalizados en vía de desarrollo, en tanto y en cuanto su aplicación sea parte de una economía controlada por Occidente. Existe, además, el peligro de que si se concibe el desarrollo desde esta óptica, se desconocerían los pobres, pues como tal, este no solo no puede llegar a beneficiarlos, sino que puede llegar a empeorar su situación.

No en vano para Dower "incluso si el modelo de crecimiento que se defiende está concebido para dar prioridad al 'crecimiento de los pobres', puede cuestionarse el supuesto de que es necesario el crecimiento 'general' para que se produzca este último" (p. 380). Para citar un ejemplo que ilustre esta afirmación, "si el mundo avanza hacia la catástrofe ecológica ello se debe más al perjuicio causado por el 'hiperdesarrollo' de los países ricos y por las consecuencias de la opulenta sociedad de consumo, que por los efectos del subdesarrollo como la erosión del suelo y la desertización" (p. 381).

Correlativamente, la visión occidentalizada del crecimiento constituye una amenaza para los países pobres, donde no suelen existir los mismos estándares de regulación que en los países ricos. Aun así, a pesar de que la apertura de nuevos mercados a través de tratados de libre comercio, suele ser considerada un sinónimo de desarrollo, ciertamente no siempre lo es. Por el contrario, favorece un aumento de brechas entre los países pobres y ricos, cuyas industrias al instalarse en los primeros, y llevar a cabo actividades y procesos de producción de bienes, terminan manipulando elementos potencialmente contaminantes, deteriorando así su entorno.

Como es de suponer, esta grave situación no incumbe a la corriente institucionalista de la justicia Global ya que esta aborda -directamenteel rol de las empresas en la pobreza y el desarrollo de los países menos favorecidos, y, por otro lado, protege los interés de los países ricos. Sin 
embargo, creemos que era oportuno dejar planteado este contexto en el binomio del que nos ocupamos en este capítulo.

Al final de lo que se trata es, precisamente, que el sector industrial de estos países adopte las medidas precautorias o preventivas necesarias para evitar perjuicios en los lugares donde decidan llevar a cabo las referidas actividades, especialmente si se trata de países pobres.

Volviendo al tema que nos ocupa, se trata de un compromiso permanente que deben asumir los Estados al momento de definir estrategias o políticas que involucren la garantía y respeto de derechos y libertades protegidas. Esa garantía, debe permear todos los escenarios; con lo cual, se desplegaría un real y el efectivo goce de los derechos.

Por lo tanto, no sería necesariamente posible admitir una reducción en el índice de pobreza cuando la vivienda adecuada, el acceso a la educación y la salud, son precarios o nulos, y cuando se desconocen las problemáticas, y, a la vez, se pretenden observar los problemas desde una óptica occidental.

Prueba de esto, es que aunque la globalización aparentemente pudiese ser el impulso que requieren los países pobres para lograr un crecimiento económico sostenido, con mejores oportunidades en el acceso y disfrute de los derechos sociales; en realidad quienes tienen el poder económico no han logrado a través de sus programas de cooperación, reducir la brecha de la pobreza y la desigualdad en los países más pobres del mundo. ${ }^{8}$

\section{El respeto por la diversidad}

Al margen de lo expuesto hasta ahora sobre el novedoso enfoque propuesto por la justicia global, sobre este tema se han planteado otros enfoques de justicia que intentan dar respuestas a muchos de los interrogantes acá planteados, entre estos ¿Cómo pensar en una sociedad justa en medio de un panorama desconcertante? Al respecto diversas

8 A esta situación se suma el clamor del propio Programa de las Naciones Unidas para el Desarrollo, que en su Informe de Desarrollo Humano del año 2003, advertía con preocupación que "si bien la globalización ha resultado sistemáticamente positiva para algunas zonas del planeta, no es menos cierto que también ha dejado de lado a otras, así como a muchos grupos dentro de los países" (PNUD, 2003). 
teorías han pretendido dibujar o trazar líneas básicas, que determinen la organización de una sociedad.

Es el caso de la teoría de justicia distributiva de Rawls, la cual, pese a aproximarse al problema e intentar abordarlo con relativo éxito, presenta ciertas inconsistencias como sería la omisión de los contextos político, económico y social respecto de que las elecciones o decisiones personales de los individuos, no interfieran en la búsqueda de esa justicia.

Campbell (1974) a partir de un planteamiento asociativo de la justicia referida al principio de distribución y a la reducción de pobreza como cuestión de humanidad, propone que la justicia no sea concebida como un equivalente ni de la distribución ni de todos los principios; aunque esta pueda llegar a ser interpretada por muchos como un criterio en el cual se incluye la distribución e incluso ciertos principios sociales-morales.

En realidad lo que se busca es señalar cómo han de interpretarse y clasificarse los argumentos que llevan a hacer distinciones de las necesidades de los habitantes de países pobres, y de esta manera justificar también las distinciones de trato 9 .

Esta configuración, deberá ajustarse a lo que es realmente importante para todos los miembros de la sociedad, dándoles la posibilidad de elegir libremente ¿Cuál y cómo ha de ser la vida que decidan llevar? Es decir, respetando la diversidad y el multiculturalismo (Sen, 2005). Con ello, bastará para cimentar adecuadamente una política efectiva de justicia social y global (Fraser, 2008), en tanto se evitarían las injusticias cometidas al aplicar una norma determinada, dado que las consecuencias no serían ciertamente las mismas para unos como para otros.

Sen (2000) al tratar el utilitarismo creé que, si bien deberían maximizarse las utilidades de todos los miembros del mismo conjunto, esto

9 Coincidimos con Campbell en uno de sus argumentos para tratar este asunto, en el que se propone asimilar -ante una negación generalizada- una conexión lógica entre justicia y pobreza. Dicho de otro modo, la justicia al entenderse como la forma de dar a cada cual lo que le corresponde, puede dar lugar a interpretaciones tan erróneas como que los desfavorecidos reciben lo poco que tienen porque se lo merecen, y este quizás es el gran problema de pensar que la pobreza es cuestión de justicia (Miller, 1999; Campbell, 1974; Goodin, 1985). Por el contrario, la justicia debe ser entendida como el espíritu de los derechos, por lo tanto, la base de una nueva sociedad debe residir en tratar justamente a los nacionales de países pobres. 
no debería hacerse de tal manera que exista un tratamiento igualitario. La razón estriba en que el utilitarismo busca tratar por igual a los individuos en las pérdidas y ganancias. En tanto ser igualitario no es necesariamente una característica fundamental para la distribución de la riqueza, dadas las diferencias muy básicas que existen entre los diversos ámbitos en los que la igualdad opera.

Esta postura encuentra su fundamento en la comprensión de diversidad de Sen (2005, p. 160), dado que las diferencias que todos tenemos, a partir de circunstancias que han configurado una u otra cultura, deben ser tomadas en cuenta para no cometer injusticias. De lo contrario, enfatizamos, lo que en un principio podría ser un trato igual para todos, en realidad termina siendo desigual para quienes en su diversidad no encajen en ese tipo de tratos.

Por ende, no debe extrañar que al confrontar la justicia global con la justicia distributiva, el ideal de igualdad encuentre varias limitaciones, siendo la principal de ellas la diversidad humana, traducida en heterogeneidad. Ello, aunque no obstante Sen reconozca que "toda teoría normativa del orden social que haya resistido, con más o menos fortuna, al paso del tiempo, al parecer puede haber exigido la igualdad de algo, lo cual, en atención a esta teoría, se considera en todo caso particularmente importante." (Sen, 1992, p. 25).

En suma, una buena voluntad que propugne por un trato igual, tomando como consideración la naturaleza de los individuos y de la justicia, puede ser perjudicial, pues se estarían creando espacios de dualidad, donde una misma situación resultase altamente positiva y benéfica para un sector, pero no para el otro. Esto supone un revés en el pretendido desarrollo, en lugar de un avance. Es por ello que la diversidad, debe convertirse en un factor determinante para la construcción de una verdadera teoría de la justicia distributiva.

\section{De los deberes correlativos al deber moral de asistencia}

Los deberes de carácter correlativo surgen de la necesidad de equilibrar la distribución de recursos y bienes en favor de los países pobres (Shue, 1996). Así como la de prohibir aquellas decisiones, usualmente 
adoptadas por los países desarrollados por medio de acuerdos multilaterales que, debiendo responder a los ideales de la justicia global, no lo hacen; generando irrupciones en el orden económico y político e imposibilitando el goce efectivo del catálogo de derechos humanos de los habitantes de los países menos favorecidos.

Sobre esta perspectiva, con la adopción de instrumentos internacionales que cumplan los deberes de este tipo, se evitarían las ya referidas injusticias al aplicarse indebidamente una norma determinada. Esto implica un compromiso, es decir, un deber correlativo para que las acciones llevadas a cabo en los países pobres se alineen con el diseño y ejecución de instrumentos internacionales.

Para citar un ejemplo, de poco o nada servirían en los programas de cooperación en el ámbito alimentario en determinados países, si al mismo tiempo se implementan acuerdos comerciales en los que se importa el mismo sistema de protección de las obtenciones de vegetales que tienen los países desarrollados, sin contemplar límites y excepciones a los derechos conferidos al titular de una obtención, con el fin de garantizar la soberanía alimentaria de los habitantes que dependen del autocultivo.

Sobre el deber moral de asistencia contra el hambre, Pogge (2007, p. 13) insiste en que tanto organizaciones internacionales como países desarrollados, están vulnerando los derechos humanos al impedir el acceso de los recursos a los países pobres. ${ }^{10}$ Una situación que, más allá del ámbito jurídico, moralmente no puede permitirse.

Pero además, entendiendo que las soluciones parten de una justa distribución de oportunidades y de capacidades, resulta entonces viable consolidar una carta de derechos a través de la acción institucional, siempre

10 Mientras que Pogge (2007, p. 11-14) ya advertía cómo la pobreza configuraba una violación de los derechos humanos, incluso de forma indirecta con la omisión; Gilabert (2004, p. 544) sugiere la existencia de un orden mundial coercitivo, a través del que justifica la obligación de erradicar la pobreza extrema por parte de los países desarrollados. Esto se logra a través de acciones negativas (Cf. Dower, 1995, p. 287). Por otra parte, Gilabert (2004, p. 544-545) además parte de la premisa de un beneficio generado a partir de la toma propia de decisiones, las cuales en no pocas ocasiones afectan los intereses de los países pobres, de ahí que esta propuesta antes que concebir cargas, lo que en realidad busca es un equilibro en pro de la justicia global. 
teniendo como un norte lo que sería el del cumplimiento hacia el deber moral ejercido sobre los agentes sociales.

No es por tanto de extrañar que desde la justicia global como desde la justicia distributiva, se busquen permanentemente vías de acceso a un mínimo vital, no de bienes y recursos, sino de oportunidades que permitan disfrutar de las libertades.

Queda claro que dentro de las políticas públicas propias del deber correlativo, debe existir un enfoque hacia el desarrollo del ser humano con el fin de ampliar sus oportunidades para disfrutar de estos, y a su vez lograr una vida digna, indistintamente de la proximidad ${ }^{11}$ o empatía hacia una determinada nación. Desde tal marco, en el Informe de Desarrollo Humano del PNUD en 2005, se hizo hincapié en la libertad y la formación de las capacidades humanas, así:

El desarrollo humano consiste (...) en la ampliación de la gama de cosas que las personas pueden hacer y de aquellas que pueden ser. Las libertades y derechos individuales importan mucho, pero las personas se verán restringidas en lo que puede hacer con esa libertad si son pobres, están enfermas, son analfabetas o discriminadas. (PNUD, 2005)

11 Sobre la proximidad y su relación con la asistencia, se parte de una premisa muy válida, y es la de la estrecha conexión con las poblaciones más cercanas, e incluso con las propias. (Cf. López \& Muñoz, 2012, p. 167; Oliveira, 2014, p. 9). Aunque el argumento tuviese en el pasado cierto grado de validez, lo que nos demuestra una realidad, plasmada en la denominada aldea global, es que moralmente estamos obligados unos con otros a prestarnos asistencia indistintamente de nuestra ubicación geográfica. Por otra parte, el problema del utilitarismo negativo radica en ese futuro desarrollo, pues si en un futuro aún quedasen rezagos de este enfoque de proximidad y asistencia, las naciones que estuvieran prestas a dar su mano, condenarían a toda su región a un estancamiento, en tanto es de entender que con el mejoramiento de las condiciones de vida, en aras de un máximo bienestar, es que el desarrollo encuentra su punto de partida. O dicho en otras palabras, a mayor bienestar, mayor poder adquisitivo, y a mayor poder adquisitivo, mayor desarrollo. En relación a si el utilitarismo negativo fuese la vía por tomar, tendríamos no sólo que apartarnos del mejoramiento de la calidad de vida de los ciudadanos de los países pobres, sino de un futuro desarrollo, ya que los esfuerzos estarían encaminados a apenas a una mejora de las condiciones mínimas vitales, evitando todo sufrimiento. (Singer, 1995; Harris, 2002) De hecho, iniciativas tales como el control de natalidad estarían bien vistas, en tanto esta doctrina propugna por la reducción del daño, y la sobrepoblación es uno de ellos. Así, lo que directa e indirectamente se lograría, es que los recursos destinados para ser compartidos entre muchos, sean repartidos de tal manera que fuesen mejor aprovechados. 
A esto se ha añadido un nuevo punto de vista que pretende dirigir el desarrollo hacia la búsqueda de soluciones para los problemas de desigualdad, discriminación y pobreza, que se encuentran estrechamente relacionados con la distribución no solo de ingresos sino de poder. (UNRISD, 2011, p. 7).

Así, comienza a extenderse una nueva dimensión del deber correlativo, con una mayor cobertura de libertades y garantías para los países pobres, en tanto las estrategias e instrumentos de cooperación, responden finalmente a un deber moral, y no a los medios ${ }^{12}$. Con lo cual, el concepto de la asistencia moral desde correlatividad se reduce a la responsabilidad compartida que tienen los países desarrollados a partir de su capacidad para erradicar toda brecha de pobreza.

Si bien es cierto que cada país debe determinar sus propias necesidades y el camino para satisfacerlas, no se puede obviar que los países desarrollados y sus ciudadanos, deben prestar asistencia en la medida de sus reales posibilidades ${ }^{13}$. En el diseño de este camino, los gobernantes de los países menos favorecidos, deben, a su vez, ser proactivos en la búsqueda de estrategias de asistencia, que permitan tratar los focos de pobreza en su contexto.

Sin embargo, la escasa preocupación de los gobernantes de los países pobres por buscar asistencia, y por realizar estudios sobre el estado actual

12 Hemos de aclarar en este punto de la discusión, que no estamos en favor de sanciones para los países que se benefician de las medidas y que repercuten de manera negativa en otro sector de la población; o sólo por el hecho de que los países pobres, sean pobres. Todo lo contrario, una sanción que sea acorde a la aplicación del principio de justicia distributiva, debe existir justamente por la adopción de estas medidas, bien porque fueron motivadas por un país rico, o bien porque han sido ejecutadas por una organización internacional.

13 Respecto a la interrogante central de lo que deberían hacer los ciudadanos de los países desarrollados: ¿Qué estoy dispuesto a dar y cuánto como asistencia a quienes lo necesitan? En un mundo en el que ni las personas ni los gobiernos aportan lo suficiente para tan sólo aliviar las necesidades vitales de los más desfavorecidos. Singer (1999, p. 62) sugiere dejar a un lado esquemas de pensamiento tales como "yo debo dar solamente una porción igual y equitativa a la de los demás", por más que este sea un argumento convincente. Con ello, se podrían llevar vidas moralmente decentes. Ahora bien, sobre el cómo y a dónde deberían ir dirigidos los aportes de los ciudadanos que decidan dar ese paso; sin duda esta es una tarea de los gobiernos elegidos democráticamente, los cuales deben trabajar en pro de diseñar e implementar proyectos de desarrollo con una vocación global, para forjar así un mejor, y cada vez más sustentable futuro para la humanidad. 
de sus habitantes (Cf. Villanueva \& Lasswell, 1992; Fernández, 2000), hace que la poca ayuda que llega a través de los programas de cooperación internacional, sea recibida como cuidados paliativos, ${ }^{14}$ puesto que, a pesar de las buenas intenciones y de la disponibilidad de recursos con que muchas veces se cuenta; la órbita de acción de dichos programas no se ajusta a una información fiable sobre las reales necesidades de estas poblaciones.

Hay que señalar que, tras la segunda guerra mundial, los recursos para la cooperación internacional eran destacables, en comparación con los destinados en años recientes, y que ya para la década de los noventa del siglo pasado habían empezado a disminuir gradualmente en un contexto de pre-recesión global (Tokatlian, \& Carvajal, 1994).

Un claro ejemplo que ilustra esta situación lo hallamos en los Objetivos de Desarrollo del Milenio ${ }^{15}$, que surgieron como una apuesta contra la reducción de la pobreza extrema, y que oprime a más de mil millones de personas en todo el mundo (PNUD, 2003). Con lo cual es válido afirmar que al día de hoy, a pesar de encontrarnos a casi un año de cumplirse el umbral establecido para el cumplimiento de las metas, estamos ante una realidad que nos demuestra como sociedad cuán lejos estamos de alcanzarlas.

Así, pues, para que el deber moral de asistencia se materialice, es un hecho ineludible que las políticas públicas se enfoquen en lograr una receptividad más ajustada de los recursos necesarios para potenciar la capacidad humana. Dicho sea de paso, las implicancias de este redireccionamiento se tendrán que ver reflejadas en un mayor compromiso hacia la toma de decisiones, y sobre cómo invertir tales recursos.

Por lo demás, el deber de asistencia y su relación con la justicia global dista del enfoque dado por Rawls sobre el papel exclusivamente asistencialista de los países favorecidos respecto de los menos favorecidos.

14 En otras ocasiones, por trabas burocráticas o por una situación de orden interno, dicha asistencia es prestada de manera tardía, sin lograr una solución a largo plazo, además de posponerse el problema, este suele persistir y a la larga, son los países pobres los que terminan revirtiendo o transformando sus necesidades.

15 Es así como el logro de los objetivos del PNUD (2005, p. 117), se ha centrado en el crecimiento económico, que sin lugar a dudas ha probado ser una herramienta para disminuir los niveles de pobreza. Sin embargo, esta estrategia funciona en cuanto a la reducción de la pobreza de ingreso, siempre que la desigualdad de ingresos inicial sea pequeña. 
Creemos, al igual que Pogge, que esos países tienen otro tipo de responsabilidades, más conscientes de las condiciones particulares de cada nación desfavorecida, así como a un orden institucional -que en realidad es un orden institucional-económico- que sea concebido en igual sentido que el orden interno.

\section{Referencias}

Altimir, O. (1981). La pobreza en América Latina: un examen de conceptos y datos. Revista de la CEPAL, 13.

Ashton, P. (1984-10-18). Poverty and its beholders. New Society, Harvard University.

Baratz, M., \& Grigsby, W. (1971) Thoughts on poverty and its elimination. Journal of Social Policy, 1(2)

Boltvini, J., \& Hernández-Laos, E. (1999). Pobreza y Distribución del Ingreso en México, Ciudad de México: Siglo XXI Editores.

Brock, G. (2017). Global Justice. En: E. Zalta (Ed.) The Stanford Encyclopedia of Philosophy. Recuperada el 10 de mayo de 2017 de: https:// plato.stanford.edu/archives/spr2017/entries/justice-global/

Campbell, T. (1974). Humanity before justice, British Journal of Political Science, 4(1), 1-16.

Cante, F. (2010). Libertades individuales y acción colectiva. Bogotá: Universidad del Rosario

Dower, N. (1995). La pobreza en el mundo. En P. Singer (Ed.). Compendio de Ética (pp. 377-390. Barcelona: Ariel.

Fernández, L. (2000). Ciudadanos precarios: la última red de protección social. Madrid: Ariel

Fraser, N. (2008). La justicia social en la era de la política de identidad: redistribución, reconocimiento y participación. Revista de Trabajo, 4(6).

Gilabert, P. (2004). The duty to eradicate global poverty: positive or negative. Ethical Theory and Moral Practice, 7.

Goodin, R. (1985). Protecting the vulnerable: a re-analysis of our social responsibilities. Chicago: University of Chicago Press. 
Grupo de Investigación en Justicia Global (2017). Diseño e implementación de estrategias para la consolidación de un grupo de investigación en justicia global [ProyectoFCSH1701T2001]. Cartagena de Indias: Universidad Tecnológica de Bolívar.

Harris, J., \& Holm, S. (2002). Extending human lifespan and the precautionary paradox. Journal of Medicine and Philosophy, 27(3).

Hine, D., \& Montiel, C. (1999). Poverty in developing nations: a cross-cultural attributional analysis. European Journal of Social Psychology, 29

López, D., \& Muñoz, F. (2012). Ayuda para el Comercio y cooperación internacional para los países de ingreso mediano: el caso de Chile. En M. Jansen, M. Sadni, \& M. Smeets (eds.) Conectarse a los mercados mundiales. Geneva: WTO Press.

Lukes, S. (1993). Five Fables about Human Rights. En S. Shute \& S. Hurley (eds.). On Human Rights. Nueva York: The Oxford Amnesty Lectures.

Mandle, J., 2006, Global Justice, Cambridge: Polity Press. Miller, D. (1999). Principles of social justice. Cambridge, MA: Harvard University Press.

Miller, D. (2007). National responsibility and global justice. Oxford University Press.

Miller, S., \& Roby, P. (1967). Poverty: changing social stratification. En P. Townsend (ed.). The concept of poverty. Londres: Heineman.

Oliveira, M. (2014). El desarrollo social en la agenda brasileña de cooperación. Buenos Aires: FLACSO.

Pinazo, D., Peris, R., \& Gámez, M. (2010). Lay beliefs about developing countries in relation to helping behaviors. The Journal of Social Psychology, 150.

Pogge, T. (2001). Priorities of global justice. Metaphilosophy, 32(1-2), 6-24.

Pogge, T. (2005). La pobreza en el mundo y los derechos humanos. Barcelona: Paladios

Pogge, T. (2007). Severe Poverty as a Human Rights Violation. En T. Pogge (ed.). Freedom from Poverty as a Human Right. Who Owes What to the Very Poor. Oxford: Oxford University Press 
Rawls, J. (1958). Justice as Fairness. Philosophical Review, 67

Rawls, J. (1982). Social unity and primary goods. En A. Sen \& B. William (eds.) Utilitarianism and Beyond. Cambridge: Cambridge University Press.

Ribotta, S. (2011). John Rawls. Sobre (des)igualdad y justicia. Madrid: Dickinson.

Risse, M., 2012, On Global Justice. Princeton: Princeton University Press.

Rist, G. (2002). Desarrollo: Historia de una creencia occidental. Madrid: Universidad Complutense de Madrid

Sen, A. (1992). Inequality Reexamined, Oxford: Oxford University Press

Sen, A. (1998). Bienestar, justicia y mercado, Barcelona: Paidós

Sen, A. (2000). Desarrollo y libertad, Buenos Aires: Editorial Planeta.

Sen, A. (2004). Elements of a Theory of Human Rights. Philosophy and Public Affairs, 32(4)

Sen, A. (2005). Human rights and capabilities. Journal of Human Development, 6(2)

Shue, H. (1996). Basic Rights: Subsistence, Affluence, and U.S. Foreign Policy. Princeton: Princeton University Press.

Singer, P. (1995). Ética práctica. Cambridge: Cambridge University Press

Singer, P. (1999-09-05). The Singer Solution to World Poverty. The New York Times Magazine, pp. 60-63

Spicker, P. (2009). Definiciones De Pobreza: doce grupos de significados. Buenos Aires: CLACSO.

Tokatlian, J., \& Carvajal, L. (1994). Tendencias de la cooperación internacional en la posguerra fría. Revista Colombia Internacional, 25.

United Nations Research Institute for Social Development. (2011). Combatir la pobreza y la desigualdad. París: UNRISD

Villanueva, L., \& Lasswell, H. (1992) El estudio de las políticas públicas. Ciudad de México: Porrúa 
